\title{
Assessment of the Calibration of Periodontal Diagnosis and Treatment Planning Among Dental Students at Three Dental Schools
}

\author{
Brittany A. Lane, DDS; Paul Luepke, DDS, MSD; Eros Chaves, DDS, MS; \\ Gerardo Maupome, BDS, MSc, PhD; George J. Eckert, MAS; \\ Steven Blanchard, DDS, MSD; Vanchit John, DDS, MSD
}

\begin{abstract}
Calibration in diagnosis and treatment planning is difficult to achieve due to variations that exist in clinical interpretation. To determine if dental faculty members are consistent in teaching how to diagnose and treat periodontal disease, variations among dental students can be evaluated. A previous study reported high variability in diagnoses and treatment plans of periodontal cases at Indiana University School of Dentistry. This study aimed to build on that one by extending the research to two additional schools: Marquette University School of Dentistry and West Virginia University School of Dentistry. Diagnosis and treatment planning by 40 third- and fourth-year dental students were assessed at each of the schools. Students were asked to select the diagnosis and treatment plans on a questionnaire pertaining to 11 cases. Their responses were compared using chisquare tests, and multirater kappa statistics were used to assess agreement between classes and between schools. Logistic regression models were used to evaluate the effects of school, class year, prior experience, and GPA/class rank on correct responses. One case had a statistically significant difference in responses between third- and fourth-year dental students. Kappas for school agreement and class agreement were low. The students from Indiana University had higher diagnosis and treatment agreements than the Marquette University students, and the Marquette students fared better than the West Virginia University students. This study can help restructure future periodontal courses for a better understanding of periodontal diagnosis and treatment planning.

Dr. Lane is in private practice in Indianapolis, IN; this study was conducted while she was a resident, Department of Periodontics and Allied Dental Programs, Indiana University School of Dentistry; Dr. Luepke is Chair, Department of Surgical Sciences, Marquette University School of Dentistry; Dr. Chaves is Chair, Department of Periodontics, University of Oklahoma School of Dentistry; Dr. Maupome is Professor, Department of Preventive and Community Dentistry, Indiana University School of Dentistry; Mr. Eckert is Biostatistician Supervisor, Department of Biostatistics, Indiana University School of Medicine; Dr. Blanchard is Director of Graduate Periodontics, Indiana University School of Dentistry; and Dr. John is Chair, Department of Periodontics and Allied Dental Programs, Indiana University School of Dentistry. Direct correspondence to Dr. Vanchit John, Department of Periodontics and Allied Dental Programs, School of Dentistry, Indiana University, 1121 West Michigan Street, Indianapolis, IN 46202; 317-274-5124; vjohn@iu.edu.
\end{abstract}

Keywords: dental education, periodontics, periodontal disease, diagnosis, treatment plan

Submitted for publication 5/2/14; accepted 7/1/14

A ccording to the most recent findings of the National Health and Nutrition Examination Survey (NHANES 2009-10), half of adults in the United States suffer from periodontitis. ${ }^{1}$ Periodontitis, a chronic inflammatory disease, causes breakdown of the supporting structures of the teeth and, if left untreated, can lead to tooth loss. Bacterial plaque biofilm has been identified as the main etiologic agent in periodontal diseases. ${ }^{2}$ A higher prevalence of periodontal disease has been associated with risk factors such as age, smoking, low socioeconomic status, and depression. ${ }^{3}$ The combination of incompletely understood risk factors and secondary etiologic agents can at times make it challenging to formulate a diagnosis for individual patients based on the current periodontal classification system.

The American Academy of Periodontology (AAP) has suggested that diagnosis and classification of periodontal diseases be based on clinical assessment. ${ }^{4}$ A complete clinical examination should include the following: 1) patient's medical, dental, social, and family history; 2) periodontal charting including probing depths and clinical attachment levels; 3 ) interpretation of radiographic data including severity and pattern of bone loss; 4) clinical signs of inflammation (bleeding on probing) and location and severity of plaque and calculus; and 5) any other relevant signs and symptoms such as pain or ulceration. ${ }^{3,4}$ 
Formulating a diagnosis and treatment plan inherently varies among clinicians as pointed out by Bader and Shugars. ${ }^{5,6}$ Those authors proposed that the clinical decision making process in dentistry be divided into three separate phases: diagnosis or the "detection phase"; decision about the appropriate intervention; and selection of treatment. Differences in the decision making process can occur in any of these phases. The diagnosis phase depends on two factors: skill in detection during the examination, and knowledge of the definition and criteria employed for identification of a disease or condition. The second phase (decision about intervention) is associated with the clinician's knowledge of the course of a given disease and present risk factors that could influence effectiveness of treatment. The third phase (treatment selection) is largely dependent on the first two phases and appropriate treatment options associated with the type of disease present. However, this phase is impacted by clinicians' personal beliefs and previous treatment outcomes.

Maupome and Sheiham ${ }^{7,8}$ and Maupome et al. ${ }^{9}$ used simulated patients to elicit diagnoses and treatment plans from dental students in the first two studies and from dental students and dentists in the third study. They concluded that strategies used to assemble diagnostically meaningful information depended on the level of complexity of the tasks at hand and on the clinical expertise of respondents. They also identified a relative overlap of diagnostic tasks and treatment planning tasks among clinicians, which evolved dynamically as the consultation progressed. These findings call for careful attention to both the difficulty of the clinical cases used in research and a careful evaluation of the developmental pathway from the novice-yet competent - clinician to the expert dental professional.

Our study sought to build on John et al. ${ }^{10}$ and to assess the level of calibration during diagnosis and treatment planning of periodontal clinical cases among dental students at three schools: Indiana University School of Dentistry (IUSD), Marquette University School of Dentistry (MUSoD), and West Virginia University School of Dentistry (WVUSD). All these schools use the same AAP Classification System to diagnose periodontal disease and subsequently propose treatment plans for patients. The aim of the study was to evaluate the level of calibration of third- and fourth-year dental students on periodontal diagnosis and treatment planning at these three dental schools. The responses of the students (across years and across schools) were contrasted against a consensus agreement arrived at by the principal investigator at IUSD and co-investigators at MUSoD and WVUSD for diagnoses and treatment plans for each case according to the AAP Classification System. Additionally, we determined if dental students' class rank and GPA were associated with superior ability to make a correct diagnosis and treatment plan.

\section{Materials and Methods}

We obtained Institutional Review Board approval (\#1212010243) at Indiana University School of Dentistry before beginning the study. We determined that, with a sample size of 20 per class year from each school, the study would have $80 \%$ power to detect a difference in the percentage of correct responses of $25 \%$ for each vignette between the third- and fourth-year students and a difference of $30 \%$ between any two schools. A slight imbalance in class sizes at WVUSD did not have a major effect on the power of the study.

We thus invited a random sample of 20 thirdand 20 fourth-year dental students from IUSD, MUSoD, and WVUSD to participate in the study via email. The class sizes at each school at the time were as follows: 104 at IUSD, 80 at MUSoD, and 50 at WVUSD. If a student was invited to participate in the study and declined, it did not affect his or her grade or evaluation in any class. Participation in the study was voluntary, and the students could withdraw at any time. The students were told that, in the study, they would view eleven cases and answer multiplechoice questions in a survey regarding the diagnosis and treatment plan for each case.

Study participants were asked to go to a designated lecture hall at IUSD, MUSoD, or WVUSD. Eleven non-identifiable unique case records were made available to participants via a PowerPoint presentation. The cases were generated by extracting existing patient information from the archived repository of case records/vignettes at IUSD. The case vignettes were collected and assembled by the principal investigator (BAL). Each case vignette included the following information: medical history, dental history, intraoral photographs, radiographs (full mouth series), and periodontal charting, which includes probing depths, clinical attachment loss, gingival recession, furcation involvement, and mobility. Eliminating all personal health information from the case vignettes masked the identity of the actual patients. 
A questionnaire was given to each participant. The first two questions asked for demographic information. Question 1 inquired about the participants' current year in dental school, and question 2 asked about their prior clinical experience (i.e., whether they had a clinical degree prior to entering dental school, such as dental hygiene) to ascertain if that background impacted periodontal diagnosis and treatment planning. Next, two questions followed each case. Questions 3 and 4 were intended to evaluate the respondents' choice of periodontal diagnosis and treatment plan for each case. Question 3 had several options for a diagnosis based on the AAP Classification System. Question 4 presented a list of procedures from which to choose for a periodontal treatment plan for each case (e.g., prophylaxis, scaling and root planing, maintenance). An optional open-ended question associated with questions 3 and 4 gave students an opportunity to enter any additional diagnoses or treatment options. The average time to assess and answer each question was approximately five minutes based on estimates of oral response times during past consensus training sessions. We estimated it would take an additional five minutes to enter the responses resulting in a total of ten minutes per vignette. Therefore, with eleven cases, a total of 110 minutes was needed to complete the questionnaire. The students were not provided with feedback or grades on this study.

The students' class rank and GPA were contrasted with their answers on the survey; this information remained confidential. The identity of students was confidential in terms of their class rank and GPA. The class rank and GPA were obtained via the following manner. First, the participant completed the two-page questionnaire on paper. The top page requested the student's name, and the second page (the questionnaire) had only an assigned study number and not the student's name. Second, the student investigator entered the student's name from the top sheet next to the subject number in an Excel file and immediately shredded the top page (leaving the questionnaire document de-identified). Third, the student investigator sent the Excel sheet to the secretary and, upon confirmation the file was received, deleted the name column of her version of the file (Excel sheet was thus de-identified). Fourth, once the secretary entered the GPA data into the Excel file, she deleted the name column and returned the updated Excel sheet to the student investigator (new version still de-identified). Finally, no document remained that connected students' names to their answers or their GPAs.

Among the students who participated in the study, there was a range of GPAs and class rank. The GPAs ranged from 4.0 (the \#1 student in class) to 2.8-3.2 for students in the middle to lower half of the class. In general, students who were in the middle ranks of their class and those who had a higher class standing participated in the study. This likely had to do with more time available for these students to participate due to their better completion of clinical graduation requirements and hence willingness to be involved in the study. The principal investigator at IUSD and co-investigators at MUSoD and WVUSD came to a consensus prior to initiation of the study for the most appropriate diagnosis and treatment plan for each case according to the AAP Classification System. These decisions served as the gold standards for diagnoses and treatment plans.

Diagnosis and treatment planning responses were tabulated by school and class year for each vignette. Multirater kappa statistics were calculated to assess the agreement for the diagnosis and treatment responses among the third- and fourth-year students at each school. The overall kappa and its standard error were used to calculate $95 \%$ Confidence Intervals as well as to compare the kappas between student groups and between schools. Comparisons between class years and schools for differences in the diagnosis and treatment planning responses for each vignette were performed using chi-square tests. Logistic regression was used to evaluate the effects of school, class year, prior experience, class rank, and GPA on correct response by vignette. Interactions among factors were examined and retained in the model when significant. Similar analyses using all vignettes combined were performed by including random effects in the logistic regression models, accounting for multiple vignettes evaluated by each subject and multiple subjects evaluating each vignette. A p-value of $<0.05$ was considered significant.

\section{Results}

A total of 120 students from the three schools participated in the study. Twenty third-year students and 20 fourth-year students from IUSD and MUSoD participated, while 23 third-year students and 17 fourth-year students from WVUSD participated (Table 1). If more than 20 students in either year volunteered to participate, the first 20 to volunteer 


\section{Table 1. School and year of participating students and their prior dental experience}

\begin{tabular}{lccc} 
& Indiana University & Marquette University & West Virginia University \\
\hline Total students & 40 & 40 & 40 \\
Third-year students & 20 & 20 & 23 \\
Fourth-year students & 20 & 20 & 17 \\
Prior dental hygiene experience & 0 & 0 & 4 \\
Prior dental assisting experience & 3 & 5 & 5
\end{tabular}

were included. At WVUSD, it was difficult to get the same number of students to participate due to the smaller class size.

As for prior experience, at IUSD there were two third-year students and one fourth-year student who reported a previous dental assisting position (Table 1). At MUSoD, there were five fourth-year students who were previously dental assistants. At WVUSD, there were three third-year students and two fourthyear students with dental assisting experience, as well as three third-year students and one fourth-year student who were previously dental hygienists.

For the majority of the cases, there was not a significant difference in responses between third- and fourth-year students. Only one case had statistically significant differences in responses between students in the two years. For case 5, the third-year students were split on diagnoses between acute necrotizing ulcerative gingivitis (33\%) and gingivitis (67\%), while the fourth-year students were split on the same two diagnoses with acute necrotizing ulcerative gingivitis (16\%) and gingivitis (82\%). One student (2\%) answered localized moderate chronic periodontitis. However, the majority of responses in both class years resulted in a correct diagnosis of gingivitis. Also, the majority of third-year (86\%) and fourthyear $(84 \%)$ students chose the correct treatment modality of dental prophylaxis corresponding to this case. In cases 6,7 , and 9, the majority of students in both class years selected the correct diagnosis. For case 10 , only $10 \%$ of third-year students and $9 \%$ of fourth-year students chose the correct diagnosis. The general trend noted is that most students chose the correct treatment modality except for two cases: in case 3 , only $35 \%$ of third-year students and $53 \%$ of fourth-year students selected the correct treatment choice, and in case 6 , only $24 \%$ of third-year students and $30 \%$ of fourth-year students selected the correct option.

There were statistically significant differences in responses for diagnosis between schools for almost all of the cases (cases 1, 3, 4, 6, 7, 8, and 11). On the other hand, there were only three cases $(7,10$, and 11) with statistically significant differences in responses between schools for treatment selection.

Kappas for school agreement and for class agreement were low, with all kappas ranging between 0.32 and 0.51 (Table 2 and Table 3). Diagnosis and treatment agreements overall were lower for thirdyear students than for fourth-year students. Students from IUSD had higher diagnosis and treatment agreements than did MUSoD students, and MUSoD students had higher diagnosis and treatment agreements than did WVUSD students. At IUSD, agreement among fourth-year students was better than agreement among third-year students for diagnosis. At MUSoD, agreement among third-year students was better than agreement among fourth-year students for diagnosis. At WVUSD, agreement among fourthyear students was better than agreement among third-year students for diagnosis. Interestingly, for treatment, third-year students from each school had slightly higher agreement than fourth-year students. All comparisons in agreement were statistically significant except for MUSoD vs. WVUSD on diagnosis and the treatment agreement between third- and fourth-year students at each school.

For cases 1 and 5, class rank and GPA had a significant impact on correct treatment response. For case 4 , prior experience had a significant impact on selecting the correct treatment response. A previous experience of dental assisting had the greatest impact, followed by no previous experience and then dental hygiene experience. For case 3, the IUSD students had more correct responses for diagnosis than the WVUSD students. Due to the limited sample size, some of the comparisons were unable to be calculated. Table 4 shows ANOVA by type. Only effect of school had a significant impact on the correct response for treatment. Table 5 shows odds ratios. The MUSoD students had more correct responses for treatment than the WVUSD students. 
Table 2. Multirater kappa statistics on diagnosis and treatment: total students and students by year for all three schools and for each school

\begin{tabular}{|c|c|c|c|c|c|}
\hline & School/Year & & Kappa & Standard Error & 95\% Confidence Interval \\
\hline Diagnosis & All three schools total & & 0.34 & 0.0015 & $0.34,0.34$ \\
\hline Diagnosis & All 3rd-year students & & 0.32 & 0.0028 & $0.31,0.33$ \\
\hline Diagnosis & All 4th-year students & & 0.36 & 0.0031 & $0.36,0.37$ \\
\hline \multirow[t]{3}{*}{ Diagnosis } & \multirow[t]{3}{*}{ Indiana University } & 3 rd year & 0.33 & 0.0090 & $0.32,0.35$ \\
\hline & & 4th year & 0.51 & 0.0087 & $0.50,0.53$ \\
\hline & & Total & 0.42 & 0.0043 & $0.41,0.42$ \\
\hline \multirow[t]{3}{*}{ Diagnosis } & \multirow[t]{3}{*}{ Marquette University } & 3 rd year & 0.36 & 0.0090 & $0.34,0.38$ \\
\hline & & 4th year & 0.32 & 0.0091 & $0.30,0.33$ \\
\hline & & Total & 0.34 & 0.0044 & $0.33,0.34$ \\
\hline \multirow[t]{3}{*}{ Diagnosis } & \multirow[t]{3}{*}{ West Virginia University } & 3 rd year & 0.32 & 0.0084 & $0.31,0.34$ \\
\hline & & 4th year & 0.37 & 0.0116 & $0.35,0.40$ \\
\hline & & Total & 0.33 & 0.0048 & $0.32,0.34$ \\
\hline Treatment & All three schools total & & 0.39 & 0.0023 & $0.38,0.39$ \\
\hline Treatment & All 3rd-year students & & 0.38 & 0.0045 & $0.37,0.39$ \\
\hline Treatment & All 4th-year students & & 0.40 & 0.0050 & $0.39,0.41$ \\
\hline \multirow[t]{3}{*}{ Treatment } & \multirow[t]{3}{*}{ Indiana University } & 3 rd year & 0.48 & 0.0164 & $0.45,0.51$ \\
\hline & & 4th year & 0.46 & 0.0145 & $0.43,0.49$ \\
\hline & & Total & 0.46 & 0.0075 & $0.45,0.47$ \\
\hline \multirow[t]{3}{*}{ Treatment } & \multirow[t]{3}{*}{ Marquette University } & $3 \mathrm{rd}$ year & 0.42 & 0.0140 & $0.40,0.45$ \\
\hline & & 4 th year & 0.40 & 0.0141 & $0.37,0.43$ \\
\hline & & Total & 0.41 & 0.0069 & $0.40,0.42$ \\
\hline \multirow[t]{3}{*}{ Treatment } & \multirow[t]{3}{*}{ West Virginia University } & 3 rd year & 0.38 & 0.0119 & $0.36,0.40$ \\
\hline & & 4th year & 0.37 & 0.0175 & $0.34,0.41$ \\
\hline & & Total & 0.37 & 0.0070 & $0.35,0.38$ \\
\hline
\end{tabular}

Table 3. Overall kappa comparisons of students' diagnosis and treatment, by school and for all three schools

\begin{tabular}{lcc} 
& Comparison & p-value \\
\hline Diagnosis & IU: 3rd year vs. 4th year & $<0.0001^{*}$ \\
MU: 3rd year vs. 4th year & $0.0018^{*}$ \\
WV: 3rd year vs. 4th year & $0.0005^{*}$ \\
Overall: 3rd year vs. 4th year & $<0.0001^{*}$ \\
School: IU vs. MU & $<0.0001^{*}$ \\
School: IU vs. WV & $<0.0001^{*}$ \\
School: MU vs. WV & 0.1246 \\
IU: 3rd year vs. 4th year & 0.3609 \\
MU: 3rd year vs. 4th year & 0.3142 \\
Treatment & Overall: 3rd year vs. 4th year & 0.6365 \\
& School: IU vs. MU & $0.0029^{*}$ \\
School: IU vs. WV & $<0.0001^{*}$ \\
School: MU vs. WV & $<0.0001^{*}$ \\
*Statistically significant at $<0.05$ & $<0.0001^{*}$ \\
\hline
\end{tabular}




\begin{tabular}{|c|c|c|c|c|c|}
\hline & Effect & Num DF & Den DF & F Value & p-value \\
\hline \multirow[t]{5}{*}{ Diagnosis } & School & 2 & 1189 & 1.35 & 0.2594 \\
\hline & Status & 1 & 1189 & 0.07 & 0.7933 \\
\hline & Prior experience & 2 & 1189 & 1.08 & 0.3383 \\
\hline & Class rank & 1 & 1189 & 1.12 & 0.2897 \\
\hline & GPA & 1 & 1189 & 1.99 & 0.1589 \\
\hline \multirow[t]{5}{*}{ Treatment } & School & 2 & 1189 & 5.67 & 0.0035 \\
\hline & Status & 1 & 1189 & 0.13 & 0.7143 \\
\hline & Prior experience & 2 & 1189 & 0.25 & 0.7826 \\
\hline & Class rank & 1 & 1189 & 0.44 & 0.5062 \\
\hline & GPA & 1 & 1189 & 0.82 & 0.3664 \\
\hline
\end{tabular}

\section{Table 5. Odds ratios by type}

\begin{tabular}{|c|c|c|c|c|}
\hline & Comparison & Estimate & Lower Limit & Upper Limit \\
\hline \multirow[t]{7}{*}{ Diagnosis } & School: IU \& WV n.s. & 1.362 & 0.892 & 2.079 \\
\hline & School: MU \& WV n.s. & 1.021 & 0.742 & 1.404 \\
\hline & School: IU \& MU n.s. & 1.334 & 0.929 & 1.915 \\
\hline & Status: $3 r d$ yr \& 4th yr n.s. & 1.041 & 0.769 & 1.409 \\
\hline & Prior experience: DentAsst \& None n.s. & 1.130 & 0.747 & 1.711 \\
\hline & Prior experience: DentHyg \& None n.s. & 1.696 & 0.807 & 3.564 \\
\hline & Prior experience: DentAsst \& DentHyg n.s. & 0.667 & 0.293 & 1.516 \\
\hline \multirow[t]{7}{*}{ Treatment } & School: IU \& WV n.s. & 1.406 & 0.875 & 2.259 \\
\hline & School: MU > WV & 1.887 & 1.299 & 2.741 \\
\hline & School: IU \& MU n.s. & 0.745 & 0.491 & 1.131 \\
\hline & Status: $3 r d$ yr \& 4th yr n.s. & 0.938 & 0.664 & 1.325 \\
\hline & Prior experience: DentAsst \& None n.s. & 1.092 & 0.681 & 1.749 \\
\hline & Prior experience: DentHyg \& None n.s. & 1.288 & 0.582 & 2.854 \\
\hline & Prior experience: DentAsst \& DentHyg n.s. & 0.847 & 0.348 & 2.065 \\
\hline
\end{tabular}

$\mathrm{IU}=$ Indiana University; $\mathrm{MU}=$ Marquette University; WV=West Virginia University; n.s.=non-significant

\section{Discussion}

No previous studies have investigated the calibration of periodontal diagnosis and treatment planning among dental students at three dental schools. All the schools in our study (IUSD, MUSoD, and WVUSD) used the same classification system, so it was assumed that there would be a level of agreement between the schools. Diagnosis is paramount because it is intended to be a summary statement that represents a thoughtful analysis of all gathered information. While Armitage and Cullinan ${ }^{11}$ suggested that practitioners should not argue over a diagnosis if the proposed treatment will be the same regardless of what the condition is labeled, there are reasons why practitioners should come to a consensus on a diagnosis. It is the diagnosis that serves as the basis for determining the most appropriate treatment plan for the patient. An accurate diagnosis is also important for communication among clinicians, between clinicians and patients, and between clinicians and insurance companies.

Learning how to interpret clinical data and to formulate a diagnosis and treatment plan are essential skills that clinicians develop during their training in dental schools. While schools use the same AAP Classification System, several reports have documented agreement inconsistencies and high variability in clinical decision making among dental faculty members. ${ }^{12-16}$ Lanning et al. ${ }^{17}$ found variation between preventive and periodontal faculty members and among periodontal graduate students in interpreting clinical findings, periodontal diagnoses, and treatment planning. When faculty members are not consistent with the clinical decision making process, 
it results in confusion for the student and perhaps delayed acquisition of appropriate clinical skills. Consequences of these variations in clinical decisions include under- or overestimation of a disease, resulting in an inappropriate basis for selecting the most suitable treatment. It is accordingly very important that faculty members responsible for guiding students through a clinical exam and then assessment of the information gathered are well calibrated. Calibration is a process designed to demonstrate that standardization has been achieved. ${ }^{18}$ From the standpoint of dental education, faculty calibration is an important process that enhances the pedagogical foundation supporting students' learning abilities and acquisition of competence. Although there is sparse research investigating calibration among dental students in the context of periodontal diagnosis and treatment planning, some studies ${ }^{17-19}$ have found that considerable controversy exists in the diagnosis and treatment planning of periodontal disease.

John et al. ${ }^{10}$ compared the calibration of predoctoral periodontal faculty members as well as of third- and fourth-year dental students at IUSD using web-based clinical periodontal case presentations. Those researchers found that agreement for diagnosis and treatment planning was overall low for dental students and periodontal faculty members, but that calibration among fourth-year students was better than for third-year students.

Calibration has been a key point of interest at IUSD especially in the Department of Periodontics. Monthly consensus training meetings are held for all periodontal faculty members with the goal of maintaining consistency while educating predoctoral students. In John et al., ${ }^{10}$ responses for diagnoses and treatment planning of periodontal cases were compared between dental students and periodontal faculty members. That study found agreement to be relatively low $(0.35-0.54)$, which is very similar to what we found in our study between classes and schools, with all kappa coefficients ranging between 0.32 and 0.51 . It is relevant to note that overall agreement was highest for IUSD for both diagnosis and treatment plan $(0.42,0.46$, respectively), followed by MUSoD $(0.34,0.41)$ and then WVUSD $(0.33$, 0.37 ). Equally important to point out is that the cases used were from the IUSD case files. Although none of these cases were presented in regular lectures for the dental students in our study, some of them may have been used for clinical rounds, meaning that we cannot rule out the possibility of some degree of case familiarity for some cases. Also, IUSD is currently the only school out of the three examined in this study to hold calibration meetings for periodontal faculty. These training sessions may have accounted for the overall better calibration of IUSD students. These possible reasons for variation among schools may be considered limitations of the study.

For all three schools, there was more agreement about treatment plans than diagnoses. John et al. ${ }^{10}$ also found that agreement among fourth-year students was better than agreement among third-year students for both diagnoses and treatment plans. Similar to their findings, we found that third-year students had lower agreement than fourth-year students at IUSD and WVUSD, whereas third-year students at MUSoD had higher agreement than fourth-year students ( 0.36 vs. 0.32 , respectively). We would typically expect third-year students to have a lower agreement than fourth-year students due to the thirdyear class's lack of clinical experience in treating periodontal patients in a clinical setting. The difference seen at MUSoD may have been due to a change in the curriculum that affected the third-year but not the fourth-year students. At MUSoD, a refresher course (three lectures) for the case-based exam for the third-year students was held in close proximity to this study. Prior to this change, most of the didactic periodontal lectures were front-loaded in the curriculum in the first two years. These results for the third-year students at MUSoD illustrate that changing a few aspects of the curriculum can be advantageous and should serve as a guide to further improvement in calibration of periodontal diagnosis and treatment planning. Another consideration is that at IUSD all didactic and clinical teaching is done by periodontists and residents, while at MUSoD didactic teaching is done by periodontists and clinical teaching is done by periodontists and dental hygienists. At WVUSD, the didactic teaching and third-year clinical teaching are done by periodontists, while the fourth-year clinical teaching is done by general practice faculty members. Whether these differences had an impact on the study results is speculative at this point, but may be seen as another possible limitation in interpreting our results.

In this study, we also looked at whether or not students were selecting the correct diagnosis and treatment plan for each of the eleven cases. The consensus choice for diagnosis and treatment plan corresponding to each case was arrived at by the principal investigator and co-investigators at each school. These investigators, who are board-certified periodontists, arrived at the consensus agreement following a face-to-face review of each case. These 
selections were made prior to initiation of the study. If we look at the percentage of correct responses of third- and fourth-year students at all three schools (Table 2), it is apparent that the percentage of correct responses was in general much lower for diagnosis than for treatment plan. The same trend was observed in responses for diagnosis between schools for the majority of the cases, but was only found in three cases $(7,10$, and 11) for treatment plan. Additionally, the majority of treatment choices were correct.

The careful clinical exercise using vignettes required considerable time for the students to go through the information. It is unclear what impact this lengthy effort may have had on the results and whether shorter exercises, broken down into smaller segments, may have led to different results. This is a methodological consideration that may have had some impact on study results, not amenable to quantification at present time. Future research will address these matters.

The case vignettes used in this study had a wide variety of clinical presentations. There were cases of gingivitis, localized aggressive periodontitis, and generalized aggressive periodontitis, in addition to various severities of chronic periodontitis. Case 3 was a generalized aggressive periodontitis case with the treatment modality of a combination of scaling and root planing with systemic antimicrobials. Although about half of the students chose the correct diagnosis ( $49 \%$ for the third years, $56 \%$ for the fourth years), it appeared that they were split between that and generalized severe chronic periodontitis (30\% for the third years, $30 \%$ for the fourth years). It is encouraging to note that the students correctly identified that both cases were a form of periodontitis because of the presence of bone loss. For a chronic periodontitis patient, the treatment is usually scaling and root planing alone. However, in the case of aggressive periodontitis, there is also a need for antimicrobials, preferably systemic antimicrobials according to the AAP position paper on this topic. ${ }^{20}$ The difference of adding an antimicrobial to the treatment plan for an aggressive case may seem like a small task, but if the clinician is unaware of the difference in etiology and pathogenesis between the two disease entities, the treatment and management are less likely to be successful.

Another area worthy of discussion is case 10 . This case presented a patient with uncontrolled Type 2 diabetes, and the correct periodontal diagnosis was generalized moderate chronic periodontitis - which only $10 \%$ of third-year students and $9 \%$ of fourth- year students correctly identified. The majority of students chose periodontitis as a manifestation of a systemic disease for the diagnosis of this case. According to the AAP Classification System, if an individual has a systemic disease that can profoundly modify the initiation and clinical course of periodontal infections, it should be included in this category. Examples include genetic disorders involving neutrophil dysfunction, immunosuppressed individuals, and viral infections. According to the current classification system, however, diabetes mellitus is not listed under this category. This can be considered one of the limitations of the current classification system.

We also looked at each student's class rank and GPA to determine if academic standing would enhance the ability to choose the correct diagnosis and treatment plan. There were only two cases (1 and 5) for which there was a significant relationship between GPA and class rank and correct treatment response. Case 1 was a generalized moderate chronic periodontitis case with the correct treatment choice of scaling and root planing. Case 5 was a gingivitis case with the correct treatment response of prophylaxis. In both cases, however, the majority of students selected the correct treatment choice. If class rank and GPA significantly affected the correct diagnosis of case 10 (discussed above) or for treatment of cases 3 or 6 , it would appear to be more significant due to the fact that the majority of students did not select the correct choice for these cases. It appears safe to say that, in general, academic standing did not affect performance. This definitely rules out the generally accepted concept that higher achieving students are more likely to answer the questions correctly. On the contrary, it demonstrates that a deeper question is at hand. Is there so much ambiguity associated with the diagnosis of periodontal disease that even the smartest students cannot determine a proper diagnosis? Or is it that the delivery of information is inconsistent?

Perhaps the answer is neither. Periodontitis is a multifaceted disease that affects different patients in different ways depending on systemic health, lifestyle, and genetics. It is not possible to standardize all periodontal cases. In our study, we found that even when diagnosis was incorrect, the proper treatment was selected the majority of the time. Also, there was more agreement between students and schools on treatment than on diagnosis. This idea cannot apply to all cases, however, because a lack of proper diagnosis, as discussed in case 3 , can affect treatment success when dealing with different disease entities that at first glance may appear to be identical. 
This study did shed some light on areas of weakness that call for improvement. This is especially true when it comes to the importance of understanding the differences in clinical presentation including rates of progression between aggressive periodontitis ${ }^{20}$ versus severe chronic periodontitis and subsequent treatment options. Going forward, efforts should be made to include more case-based teaching in order to simulate how students will be working through cases after they graduate and are practicing without the guidance of experienced faculty. Rather than having rigid diagnosis categories as the focal point, students should focus on etiology and clinical disease presentation and consider the best way to treat their individual patients based on all information in a given case.

\section{Conclusion}

In our study, we set out to determine if thirdand fourth-year dental students at three dental schools were calibrated in terms of periodontal diagnosis and treatment planning. We expected some variability and found that agreement between schools and students was relatively low. We also looked at the number of correct responses for each case. Interestingly, students from all schools had higher percentages of correct responses for treatment choice than for diagnosis. Class rank and GPA did not play a significant role overall in selecting the proper diagnosis or treatment plan. This study highlighted some areas of weakness that can now be emphasized in future periodontal courses and lectures for a better understanding of periodontal diagnosis and treatment planning. Implementing a consensus training program for faculty and making adjustments to the curriculum to provide case-based learning situations closer to the time of clinical training may enhance understanding and agreement on diagnosis and treatment planning for dental students.

\section{REFERENCES}

1. Eke PI, Dye BA, Wei L, et al. Prevalence of periodontitis in adults in the United States: 2009 and 2010. J Dent Res 2012;91(10):914-20.
2. Fleming T. Periodontitis. Ann Periodontol 1999;4:32-7.

3. Pihlstrom BL. Periodontal risk assessment, diagnosis, and treatment planning. Periodontol 2000 2001;25:37-58.

4. Armitage GC. Diagnosis of periodontal diseases. J Periodontol 2003;4(8):1237-47.

5. Bader J, Shugars D. Understanding dentists' restorative treatment decisions. J Public Health Dent 1992;52:102-10.

6. Bader J, Shugars D. Need for change in standards of caries diagnosis: epidemiology and health services research perspective. J Dent Educ 1993;57:415-21.

7. Maupome G, Sheiham A. Clinical decision making in restorative dentistry: content analysis of diagnostic thinking processes and concurrent concepts used in an educational environment. Eur J Dent Educ 2000;4(4):143-52.

8. Maupome G, Sheiham A. Explanatory models in the interpretations of clinical features of dental patients within a university dental education setting. Eur J Dent Educ 2002;6(1):2-8.

9. Maupome G, Schrader S, Mannan S, et al. Diagnostic thinking and information used in clinical decision making: a qualitative study of expert and student dental clinician. BMC Oral Health 2010;10(1):11.

10. John V, Lee SJ, Prakasam S, et al. Consensus training: an effective tool to minimize variations in periodontal diagnosis and treatment planning among faculty and students. J Dent Educ 2012;77(8):1022-32.

11. Armitage G, Cullinan M. Comparison of the clinical features of chronic and aggressive periodontitis. Periodontol 2000 2010;53:12-27.

12. Bader JD, Shugars DA. Variation in dentists' clinical decisions. J Public Health Dent 1995;55(3):181-8.

13. Bader JD, Shugars DA. Agreement among dentists' recommendations for restorative treatment. J Dent Res 1993;72(5):891-6.

14. Scruggs RR. Effects of specific criteria and calibration on examiner reliability. J Dent Hyg 1989;63(3):125-9.

15. Garland KV, Newell KJ. Dental hygiene faculty calibration in the evaluation of calculus detection. J Dent Educ 2009;73(3):383-9.

16. O'Connor P, Lorey RE. Improving interrater agreement in evaluation in dentistry by the use of comparison stimuli. J Dent Educ 1978;42(4):174-9.

17. Lanning SK, Pelok SD, Williams BC, et al. Variation in periodontal diagnosis and treatment planning among clinical instructors. J Dent Educ 2005;69(3):325-37.

18. Courts F. Standardization and calibration in the evaluation of clinical performance. J Dent Educ 1997;61(12):947-9.

19. Baelum V, Lopez R. Defining and classifying periodontitis: need for a paradigm shift? Eur J Oral Sci 2003;111(1):2-6.

20. Parameter on aggressive periodontitis. J Periodontol 2000;71:867-9. 